\title{
Histologic purity of signet ring cell carcinoma is a favorable risk factor for lymph node metastasis in poorly cohesive, submucosa- invasive early gastric carcinoma
}

\author{
Yon Hee Kim¹ - Ji Hye Park ${ }^{2}$ Cheol Keun Park ${ }^{2}$ Jie-Hyun Kim³ \\ Sang Kil Lee ${ }^{4}$ Yong Chan Lee ${ }^{4}$ Sung Hoon $\mathrm{Noh}^{5} \cdot$ Hyunki Kim² $^{2}$
}

Received: 1 July 2016/Accepted: 12 September 2016/Published online: 23 September 2016

(c) The International Gastric Cancer Association and The Japanese Gastric Cancer Association 2016

\begin{abstract}
Background The prediction of biologic behavior of poorly cohesive early gastric carcinoma (EGC) is an important issue in the selection of the treatment modality. To elucidate the risk factors for lymph node metastasis (LNM) of poorly cohesive EGC, we focused on the histologic purity of the poorly cohesive component and evaluated the impact of this factor on LNM.

Methods We divided poorly cohesive EGC into (1) pure signet ring cell (SRC) carcinoma, which was defined as composed only of signet ring cells or poorly cohesive cells and (2) mixed SRC carcinoma, defined as poorly cohesive carcinoma with minor tubular components. We reviewed the clinicopathologic features, including age, sex, location, size, depth, lymphovascular invasion (LVI), LNM, ulceration, and intestinal metaplasia between the two groups in a
\end{abstract}

Electronic supplementary material The online version of this article (doi:10.1007/s10120-016-0645-x) contains supplementary material, which is available to authorized users.

Hyunki Kim

kimhyunki@yuhs.ac

1 Department of Pathology, Ajou University School of Medicine, Suwon, Republic of Korea

2 Department of Pathology, Yonsei University College of Medicine, 50 Yonsei-ro, Seodaemun-gu, Seoul 120-752, Republic of Korea

3 Department of Internal Medicine, Gangnam Severance Hospital, Yonsei University College of Medicine, Seoul, Republic of Korea

4 Division of Gastroenterology, Department of Internal Medicine, Yonsei University College of Medicine, Seoul, Republic of Korea

5 Department of Surgery, Yonsei University College of Medicine, Seoul, Republic of Korea large series of poorly cohesive, submucosa-invasive EGC $(n=317)$.

Results LNM was found in 58 cases (18.3\%). Mixed SRC carcinoma histologic type $(p<0.001)$, larger tumor size (more than $2 \mathrm{~cm})(p=0.012)$, and the presence of LVI $(p<0.001)$ were associated with LNM. Pure SRC carcinomas accounted for $56.2 \%(178 / 317)$ of the cases. Fourteen pure SRC carcinomas $(7.8 \%)$ showed LNM, whereas 44 mixed SRC carcinomas $(31.9 \%)$ exhibited LNM $(p<0.001)$. On multivariate logistic regression, the presence of LVI (odds ratio 6.737; $95 \%$ confidence interval 2.714-16.720; $p<0.001)$ and mixed SRC carcinoma histologic type (odds ratio 4.674; $95 \%$ confidence interval 2.370-9.216; $p<0.001$ ) were independent predictors of LNM in poorly cohesive, submucosa-invasive EGC.

Conclusions The presence of a tubular component in SRC carcinoma was a risk factor for LNM in poorly cohesive, submucosa-invasive EGC. On the basis of this finding, we propose that the presence of a minor tubular component or the purity of the poorly cohesive/SRC carcinoma component should be reported in daily pathologic practice.

Keywords Early gastric carcinoma - Pure signet ring cell carcinoma $\cdot$ Lymph node metastasis

\section{Introduction}

Technical advances in endoscopic treatment of early gastric carcinoma (EGC) have created unprecedented opportunities to treat EGC patients. In particular, endoscopic submucosal dissection (ESD) technology overcomes the limitation of tumor size and makes possible successful en bloc resection $[1,2]$. This endoscopic approach prevents 
unnecessary gastrectomy for EGC that is expected to have an extremely low probability of lymph node metastasis (LNM) and facilitates organ preservation, which is an important aspect of quality of life [1]. Thus, the number of EGC patients who receive endoscopic mucosal resection or ESD has risen rapidly and steadily [3, 4]. This rapid increase in the number of patients treated by endoscopic mucosal resection or ESD highlights the importance of a precise pathologic risk assessment of LNM in resected specimens. However, there are no conclusive guidelines for undifferentiated adenocarcinoma, which accounts for nearly half of EGC. The currently accepted ESD indications for EGC, based on a proposal by Gotoda et al. [5] are (1) an intramucosal, nonulcerative differentiated adenocarcinoma, regardless of size, (2) an ulcerative intramucosal differentiated adenocarcinoma of size $3.0 \mathrm{~cm}$ or smaller, and (3) a differentiated adenocarcinoma of size $3.0 \mathrm{~cm}$ or smaller with minute submucosal invasion (500 $\mu \mathrm{m}$ or less). A small $(2.0 \mathrm{~cm}$ or less) intramucosal undifferentiated adenocarcinoma without ulceration is a marginal indication for ESD. Although Hirasawa et al. [6] provided robust evidence that there was no risk of LNM in patients with undifferentiated EGC fulfilling the expanded criteria, the safety of the endoscopic approach for an undifferentiated cancer, such as signet ring cell (SRC) carcinoma or poorly cohesive adenocarcinoma, has been controversial and inconclusive.

SRC carcinoma is defined as a tumor that predominantly consists of isolated or small groups of tumor cells containing intracytoplasmic mucin [7]. SRC carcinoma accounts for 16.8-35.5\% of gastric cancer cases [8-11]. The tumor cells of SRC carcinoma show several morphologic variants, including (1) classic SRC carcinomas, characterized by intracytoplasmic mucin vacuoles with eccentric nuclei, (2) tumor with central nuclei resembling histiocytes, (3) tumor with deeply eosinophilic cytoplasm, (4) small cells with scant mucin, and (5) anaplastic cells with scant mucin [7]. The behavior of SRC carcinoma in EGC is controversial. The behavior in early-stage SRC carcinoma has been reported to be alternately more favorable than $[9,11-13]$ or equivalent to $[14,15]$ other types. We recently found a more favorable prognosis for SRC carcinoma than the differentiated type in the early stage, in contrast with a worse prognosis for advanced SRC carcinoma [16]. In terms of tumor histology in EGC, we also reported different LNM rates among pure tubular adenocarcinoma, tubular adenocarcinoma with a minor SRC carcinoma component (mixed type), and SRC carcinoma [11]. Mixed-type EGC was more aggressive than pure tubular adenocarcinoma or SRC carcinoma. The LNM rate of SRC carcinoma was comparable to that of pure tubular adenocarcinoma [11]. There are similar studies showing the aggressiveness of tubular adenocarcinoma with a minor SRC carcinoma component in EGCs [17, 18]. By definition, the proportion of isolated cancer cells of SRC carcinoma should be greater than $50 \%$ in a tumor [7, 19], That is, SRC carcinoma encompassing a minor component of differentiated adenocarcinoma is also diagnosed as SRC carcinoma. The behavioral significance of such a minor tubular component in SRC carcinoma has not been addressed. In particular, the differences in LNM rate between pure SRC carcinoma and SRC carcinoma with a minor tubular component remain to be elucidated. Considering the worse behaviors of mixed-type differentiated EGCs observed in our previous studies [11], there might also be differences in behavior between pure SRC carcinoma and SRC carcinoma with minor tubular components (mixed SRC carcinoma). To address this question, we evaluated the clinicopathologic features of a large series of poorly cohesive, submucosa-invasive EGCs with focus on the purity of the SRC carcinoma component. For further evaluation of histogenetic differences between the two groups, we compared phenotypic differences using lineageassociated markers identified by immunohistochemistry (IHC).

\section{Materials and methods}

\section{Patients and clinicopathologic evaluation}

Three hundred seventeen patients who underwent gastrectomy with lymph node dissection at Severance Hospital and in whom poorly cohesive, submucosa-invasive EGC was diagnosed from January 2010 to December 2012 were included. The following EGC cases were excluded: cancers treated with neoadjuvant chemotherapy, multiple gastric cancers, and cancers of remnant stomach. Clinical and pathologic parameters were evaluated, including age, sex, location of tumor, size, depth of invasion, lymphovascular invasion (LVI), LNM, ulceration, state of Helicobacter pylori infection, and intestinal metaplasia in the adjacent nonneoplastic mucosa. The depth of invasion was defined as the distance from the lowest level of the muscularis mucosae (or surface of ulceration) to the end of the deepest tumor invasion. The level of submucosal invasion was divided into three sublevels (upper third, SM1; middle third, SM2; and lower third, SM3). Ulceration was defined histologically as a disruption of the muscularis mucosae with or without granulation tissue formation or submucosal fibrosis. Information on $H$. pylori infection was collected from medical records. To assess the purity of the SRC carcinoma component, we reviewed archival hematoxylin and eosin stained slides of all cases and divided the poorly cohesive, submucosa-invasive EGCs into (1) pure SRC carcinoma histologic type, which was composed of only 
SRCs or poorly cohesive tumor cells (Fig. 1a, b) and (2) mixed SRC carcinoma, which was poorly cohesive EGC showing a minor (less than $50 \%$ ) tubular or papillary component (Fig. 1c, d). The study was approved by the Institutional Review Board of Severance Hospital (approval no. 4-2014-0667).

\section{Immunohistochemistry}

Among the 317 cases, 92 diagnosed in 2012 were evaluated by IHC for lineage-associated markers, including mucin $5 \mathrm{AC}$, mucin 6 , mucin 2 and CDX2. Mucin 5AC and mucin 6 were used as markers of the gastric foveolar and antral phenotype respectively [20, 21]. Mucin 2 and CDX2 were used as markers of intestinal differentiation [20, 21]. IHC was performed with a Ventana XT automated stainer (Ventana, Tucson, AZ, USA) with use of antibodies for mucin 5AC (1:2000, clone CLH5; Novocastra, UK), mucin 6 (1:200, clone CCP58, Novocastra), mucin 2 (1:200, clone CLH2, Novocastra), and CDX2 (1:50, clone EPR2764Y, Cell Marque, Rocklin, CA, USA). Sections were deparaffinized with EZ Prep solution (Ventana). CC1 standard [pH 8.4 buffer containing tris(hydroxymethyl)aminomethane-borate-EDTA] was used for antigen retrieval and blocked with inhibitor $\mathrm{D}\left(3 \% \mathrm{H}_{2} \mathrm{O}_{2}\right)$ for 4 min at $37{ }^{\circ} \mathrm{C}$. Slides were incubated with primary antibody for $40 \mathrm{~min}$ at $37{ }^{\circ} \mathrm{C}$ followed by a universal secondary antibody for $20 \mathrm{~min}$ at $37^{\circ} \mathrm{C}$. Slides were incubated in streptavidin-horseradish peroxidase $\mathrm{D}$ for
16 min at $37{ }^{\circ} \mathrm{C}$ and then the substrate, $3,3^{\prime}$-diaminobenzidine tetrahydrochloride $\mathrm{H}_{2} \mathrm{O}_{2}$, was added for $8 \mathrm{~min}$, followed by hematoxylin and bluing reagent counterstaining at $37{ }^{\circ} \mathrm{C}$. Cases showing more than $10 \%$ immunoreactivity for each marker were identified as positive. Two pathologists (Y.H. Kim and H. Kim) assessed the IHC results blinded to all clinicopathologic information.

\section{Statistical analysis}

Data were analyzed with IBM SPSS Statistics for Windows version 23.0 (IBM, Armonk, NY, USA). The chi-squared test and Student's $t$ test were used for categorical variables and continuous variables respectively. To identify independent predictive factors of LNM, a Cox logistic regression model was fitted. Statistical significance was assumed when $P<0.05$.

\section{Results}

\section{Clinicopathologic features of poorly cohesive, submucosa-invasive EGCs according to LNM}

LNM was found in 58 cases $(18.3 \%)$. The clinicopathologic features of the 317 EGCs according to the LNM status are summarized in Table 1. Among the LNM-positive EGCs, $75.9 \%$ (44/58) of the cases were mixed SRC carcinoma histologic type. However, $63.7 \%$ (165/259) of
Fig. 1 Representative images of a case of pure signet ring cell carcinoma $(\mathbf{a} \times 40, \mathbf{b} \times 200)$ and a case of mixed signet ring cell carcinoma $(\mathbf{c} \times 40, \mathbf{d} \times 200)$
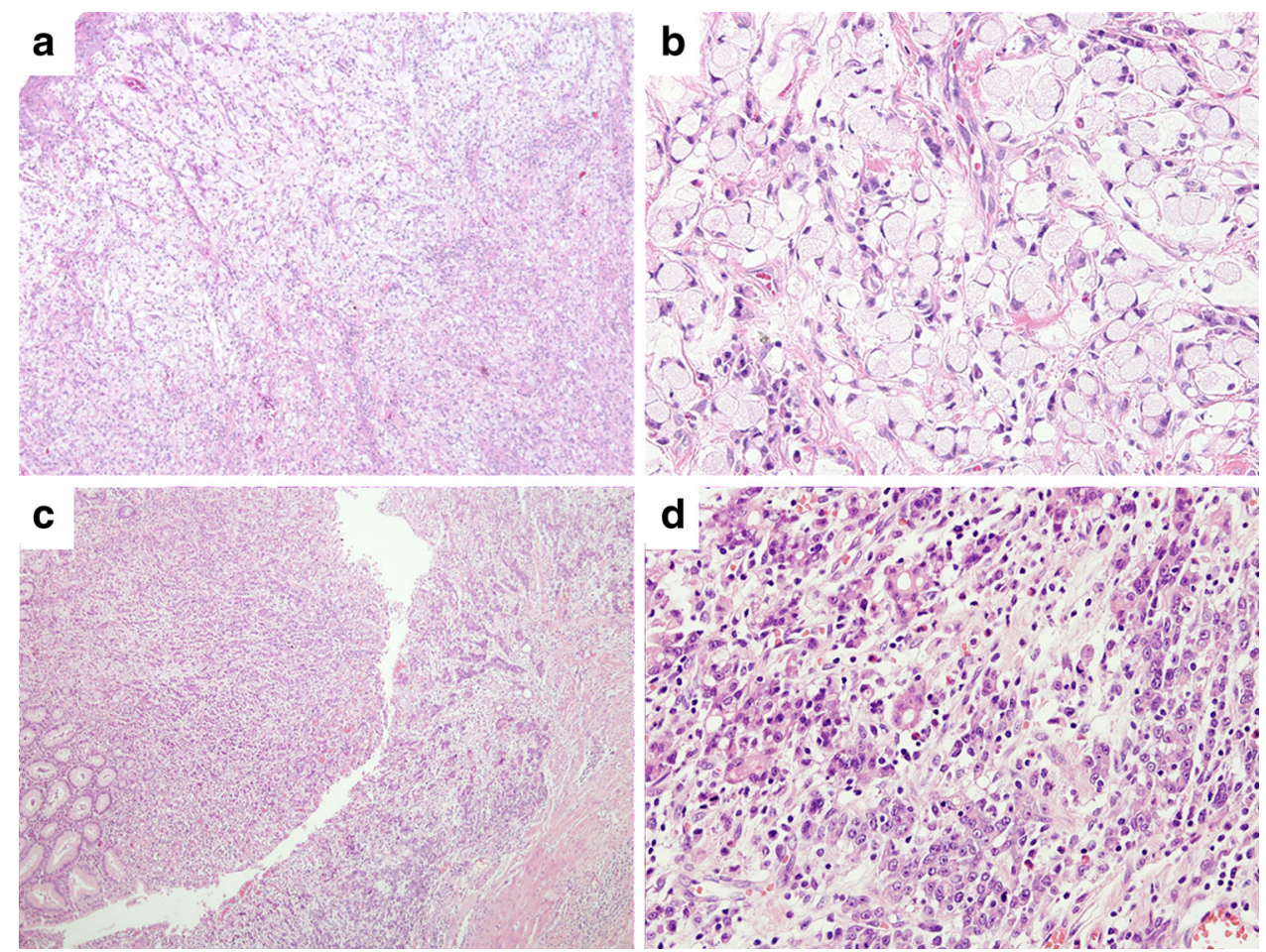
Table 1 Clinicopathologic features of poorly cohesive, submucosa-invasive early gastric carcinoma according to lymph node metastasis ( $L N M)$

\begin{tabular}{|c|c|c|c|c|}
\hline Parameters & $\begin{array}{l}\text { Total } \\
(n=317)\end{array}$ & $\begin{array}{l}\text { No LNM } \\
(n=259)\end{array}$ & $\begin{array}{l}\text { Presence of } \\
\text { LNM }(n=58)\end{array}$ & $p$ value \\
\hline Age (years) & & $55.0 \pm 11.9$ & $54.0 \pm 10.9$ & 0.561 \\
\hline Sex & & & & 0.598 \\
\hline Male & $130(41.0 \%)$ & $108(41.7 \%)$ & $22(37.9 \%)$ & \\
\hline Female & $187(59.0 \%)$ & $151(58.3 \%)$ & $36(62.1 \%)$ & \\
\hline Location & & & & 0.769 \\
\hline Upper third & $47(14.8 \%)$ & $40(15.4 \%)$ & $7(12.1 \%)$ & \\
\hline Middle third & $143(45.1 \%)$ & $115(44.4 \%)$ & $28(48.3 \%)$ & \\
\hline Lower third & $127(40.1 \%)$ & $104(40.2 \%)$ & $23(39.7 \%)$ & \\
\hline Size 1 & & & & 0.012 \\
\hline$\leq 2 \mathrm{~cm}$ & $173(54.6 \%)$ & $150(47.1 \%)$ & $23(22.4 \%)$ & \\
\hline$>2 \mathrm{~cm}$ & $144(45.4 \%)$ & $109(52.9 \%)$ & $35(77.6 \%)$ & \\
\hline Size 2 & & & & 0.025 \\
\hline$\leq 3 \mathrm{~cm}$ & $248(78.2 \%)$ & $209(80.7 \%)$ & $39(67.2 \%)$ & \\
\hline$>3 \mathrm{~cm}$ & $69(21.8 \%)$ & $50(19.3 \%)$ & $19(32.8 \%)$ & \\
\hline Invasion depth 1 & & & & 0.197 \\
\hline SM1 & $64(20.2 \%)$ & $57(22.0 \%)$ & $7(12.1 \%)$ & \\
\hline SM2 & $98(30.9 \%)$ & $80(30.9 \%)$ & $18(31.0 \%)$ & \\
\hline SM3 & $155(48.9 \%)$ & $122(47.1 \%)$ & $33(56.9 \%)$ & \\
\hline Invasion depth 2 & & & & 0.178 \\
\hline$\leq 500 \mu \mathrm{m}$ & $45(14.2 \%)$ & $40(15.4 \%)$ & $5(8.6 \%)$ & \\
\hline$>500 \mu \mathrm{m}$ & $272(85.8 \%)$ & $219(84.6 \%)$ & $53(91.4 \%)$ & \\
\hline Lymphovascular invasion & & & & $<0.001$ \\
\hline Absent & $268(84.5 \%)$ & $241(93.1 \%)$ & $27(46.6 \%)$ & \\
\hline Present & $49(15.5 \%)$ & $18(6.9 \%)$ & $31(53.4 \%)$ & \\
\hline Ulceration & & & & 0.235 \\
\hline Absent & $243(76.7 \%)$ & $202(78.0 \%)$ & $41(70.7 \%)$ & \\
\hline Present & $74(23.3 \%)$ & $57(22.0 \%)$ & $17(29.3 \%)$ & \\
\hline Helicobacter pylori infection & & & & 0.158 \\
\hline Absent & $92(53.5 \%)$ & $77(56.2 \%)$ & $15(42.9 \%)$ & \\
\hline Present & $80(46.5 \%)$ & $60(43.8 \%)$ & $20(57.1 \%)$ & \\
\hline Intestinal metaplasia & & & & 0.035 \\
\hline Absent & $160(50.5 \%)$ & $138(53.3 \%)$ & $22(37.9 \%)$ & \\
\hline Present & $157(49.5 \%)$ & $121(46.7 \%)$ & $36(62.1 \%)$ & \\
\hline Histologic type & & & & $<0.001$ \\
\hline Pure SRC carcinoma & $179(56.5 \%)$ & $165(63.7 \%)$ & $14(24.1 \%)$ & \\
\hline Mixed SRC carcinoma & $138(43.5 \%)$ & $94(36.3 \%)$ & $44(75.9 \%)$ & \\
\hline
\end{tabular}

SM1 submucosal invasion of the upper third, SM2 submucosal invasion of the middle third, SM3 submucosal invasion of the lower third, SRC signet ring cell

the LNM-negative EGCs were pure SRC carcinoma histologic type $(p<0.001)$. Well-known risk factors of larger tumor size ( $2 \mathrm{~cm}$ or more) $(p=0.012)$ and the presence of LVI $(p<0.001)$ were associated with LNM. However, depth of submucosal invasion and the presence of ulceration, other well-known risk factors for LNM, were not significantly associated with LNM in our cohort.

\section{Comparison of clinicopathologic features according to the purity of SRC carcinoma component}

On histologic review, pure SRC carcinomas accounted for $56.2 \%(178 / 317)$ of the cases. LNM were found in 14 cases $(7.9 \%)$ in this group. In contrast, of 139 cases of mixed SRC carcinoma, 44 (31.7\%) included LNM 
$(p<0.001)$. In addition, pure SRC carcinoma histologic type was associated with infrequent LVI $(p<0.001)$ and lower incidence of intestinal metaplasia in the adjacent nonneoplastic mucosa $(p<0.001)$ (Table 2). There were no differences in age, sex, tumor location, tumor size, $H$. pylori infection, ulceration, and depth of submucosal invasion between the two groups.

\section{Risk factors for LNM in poorly cohesive, submucosa-invasive EGCs}

On multivariate logistic regression analysis with the three features of tumor size, LVI, and histologic purity of SRC carcinoma, which were revealed as being associated with LNM in Table 1, the presence of LVI (odds ratio 6.737;

Table 2 Clinicopathologic characteristics of poorly cohesive, submucosa-invasive early gastric carcinoma according to the purity of the signet ring cell $(S R C)$ carcinoma/poorly cohesive component

\begin{tabular}{|c|c|c|c|c|}
\hline Parameters & $\begin{array}{l}\text { Total } \\
(n=317)\end{array}$ & $\begin{array}{l}\text { Pure SRC carcinoma histologic type } \\
(n=179)\end{array}$ & $\begin{array}{l}\text { Mixed SRC carcinoma histologic type } \\
(n=138)\end{array}$ & $p$ value \\
\hline Age (years) & & $55.81 \pm 11.7$ & $53.5 \pm 11.7$ & 0.08 \\
\hline Sex & & & & 0.746 \\
\hline Male & $130(41.0 \%)$ & $72(40.2 \%)$ & $59(42.0 \%)$ & \\
\hline Female & $187(59.0 \%)$ & $107(59.8 \%)$ & $80(58.0)$ & \\
\hline Location & & & & 0.203 \\
\hline Upper third & $47(14.8 \%)$ & $31(17.3 \%)$ & $16(11.6 \%)$ & \\
\hline Middle third & $143(45.1 \%)$ & $74(41.3 \%)$ & $70(50.0 \%)$ & \\
\hline Lower third & $127(40.1 \%)$ & $74(41.3 \%)$ & $53(38.4 \%)$ & \\
\hline Size 1 & & & & 0.227 \\
\hline$\leq 2 \mathrm{~cm}$ & $173(54.6 \%)$ & $103(57.5 \%)$ & $70(50.7 \%)$ & \\
\hline$>2 \mathrm{~cm}$ & $144(45.4 \%)$ & $76(42.5 \%)$ & $68(48.3 \%)$ & \\
\hline Size 2 & & & & 0.992 \\
\hline$\leq 3 \mathrm{~cm}$ & $248(78.2 \%)$ & $140(78.2 \%)$ & $108(78.3 \%)$ & \\
\hline$>3 \mathrm{~cm}$ & $69(21.8 \%)$ & $39(21.8 \%)$ & $30(21.7 \%)$ & \\
\hline Invasion depth 1 & & & & 0.796 \\
\hline SM1 & $64(20.2 \%)$ & $34(19.0 \%)$ & $30(21.7 \%)$ & \\
\hline SM2 & $98(30.9 \%)$ & $55(30.7 \%)$ & $44(31.2 \%)$ & \\
\hline SM3 & $155(48.9 \%)$ & $90(50.3 \%)$ & $65(47.1 \%)$ & \\
\hline Invasion depth 2 & & & & 0.606 \\
\hline$\leq 500 \mu \mathrm{m}$ & $45(14.2 \%)$ & $27(15.1 \%)$ & $18(13.0 \%)$ & \\
\hline$>500 \mu \mathrm{m}$ & $272(85.8 \%)$ & $152(84.9 \%)$ & $120(87.0 \%)$ & \\
\hline Lymphovascular invasion & & & & $<0.001$ \\
\hline Absent & $268(84.5 \%)$ & $165(92.2 \%)$ & $94(68.1 \%)$ & \\
\hline Present & $49(15.5 \%)$ & $14(7.8 \%)$ & $44(31.9 \%)$ & \\
\hline Ulceration & & & & 0.08 \\
\hline Absent & $243(76.7 \%)$ & $144(80.4 \%)$ & $100(71.9 \%)$ & \\
\hline Present & $74(23.3 \%)$ & $35(19.6 \%)$ & $39(28.3 \%)$ & \\
\hline Intestinal metaplasia & & & & $<0.001$ \\
\hline Absent & $160(50.5 \%)$ & $110(61.5 \%)$ & $50(36.8 \%)$ & \\
\hline Present & $157(49.5 \%)$ & $69(38.5 \%)$ & $88(63.2 \%)$ & \\
\hline $\begin{array}{l}\text { Helicobacter pylori } \\
\text { infection }\end{array}$ & & & & 0.583 \\
\hline Absent & $92(53.5 \%)$ & $51(55.4 \%)$ & $41(51.3 \%)$ & \\
\hline Present & $80(46.5 \%)$ & $41(44.6 \%)$ & $39(48.7 \%)$ & \\
\hline Lymph node metastasis & & & & $<0.001$ \\
\hline Absent & $259(81.7 \%)$ & $165(92.2 \%)$ & $94(68.1 \%)$ & \\
\hline Present & $58(18.3 \%)$ & $14(7.8 \%)$ & $44(31.9 \%)$ & \\
\hline
\end{tabular}

SM1 submucosal invasion of the upper third, SM2 submucosal invasion of the middle third, SM3 submucosal invasion of the lower third 
$95 \%$ confidence interval 2.714-16.720; $p<0.001)$ and mixed SRC carcinoma histologic type (odds ratio 4.674; $95 \%$ confidence interval $2.370-9.216 ; p<0.001$ ) were independent predictors for LNM of poorly cohesive, submucosa-invasive EGC (Table 3).

\section{Comparison of mucin expression between pure and mixed SRC carcinoma histologic types}

To evaluate phenotypic differences between the pure and mixed SRC carcinoma groups, we performed IHC for lineage-associated markers. Among the 97 cases, mucin 5AC, mucin 6 , mucin 2 , and $\mathrm{CDX} 2$ postitivity was found in 46 cases $(50.0 \%), 43$ cases (44.3\%), 33 cases ( $35.9 \%$ ), and 86 cases $(93.5 \%)$ respectively. In the pure SRC carcinoma histologic type, positivity for the gastric mucins mucin $5 \mathrm{AC}$ and mucin 6 was found in $49 \%(26 / 53)$ and $44 \%$ (23/53) of the cases respectively. In the mixed SRC carcinoma group, positivity for the intestinal markers mucin 2

Table 3 Risk factors for lymph node metastasis in poorly cohesive, submucosa-invasive early gastric carcinoma

\begin{tabular}{llll}
\hline Parameters & Odds ratio & $95 \%$ CI & $p$ value \\
\hline $\begin{array}{l}\text { Size } 1 \\
\leq 2 \mathrm{~cm}\end{array}$ & 1.508 & $0.772-3.150$ & 0.067 \\
$\quad>2 \mathrm{~cm}$ & & & \\
$\begin{array}{l}\text { Lymphovascular invasion } \\
\quad \text { Absent }\end{array}$ & & & \\
$\quad$ Present & 3.373 & $1.198-9.498$ & $<0.001$ \\
Histologic type & & & \\
$\quad \begin{array}{l}\text { Pure SRC carcinoma } \\
\text { Mixed SRC carcinoma }\end{array}$ & 13.777 & $6.371-29.791$ & $<0.001$ \\
\hline
\end{tabular}

$C I$ confidence interval, $S R C$ signet ring cell and CDX2 was found in $41 \%(16 / 39)$ and $97 \%(38 / 39)$ of the cases respectively. However, there were no differences in positivity for any of the markers between the two groups (Table 4, Fig. S1).

\section{Discussion}

The safety and long-term efficacy of ESD for treatment of undifferentiated EGC remain controversial [22]. Because undifferentiated adenocarcinoma includes solid and nonsolid poorly differentiated SRC carcinoma and mucinous adenocarcinoma [23], undifferentiated EGC may be a histologically and prognostically heterogeneous group. With this view in mind, we observed different LNM rates among intestinal-type and diffuse-type poorly differentiated adenocarcinoma and SRC carcinoma. In the three groups, SRC carcinoma showed the lowest LNM rate $(6.3 \%, 58 / 927)$ and intestinal-type poorly differentiated adenocarcinoma had the highest rate $(15.8 \%, 29 / 183)$ [24]. Solid-type poorly differentiated carcinoma seemed to be more heterogeneous and consisted of both favorable subtypes such as Epstein-Barr virus (EBV)-associated carcinoma and unfavorable subtypes such as intestinal-type poorly differentiated adenocarcinoma. EBV-associated EGC, which frequently has an undifferentiated histologic appearance, is associated with an extremely low risk of LNM [25]. In contrast, intestinal-type poorly differentiated adenocarcinoma is more aggressive than other types of EGC [24]. In this study, we focused on poorly cohesive adenocarcinoma according to the WHO classification [26]. This type could be classified as SRC carcinoma or nonsolid-type poorly differentiated adenocarcinoma according to the Japanese classification [19]. By assessing the risk for LNM in poorly cohesive adenocarcinoma more precisely,
Table 4 Expression of lineageassociated markers according to histologic type

\begin{tabular}{lcllc}
\hline Parameters & Total $(N=92)$ & $\begin{array}{l}\text { Pure SRC carcinoma } \\
\text { histologic type }(n=53)\end{array}$ & $\begin{array}{l}\text { Mixed SRC carcinoma } \\
\text { histologic type }(n=39)\end{array}$ & $p$ value \\
\hline $\begin{array}{l}\text { Mucin } 6 \\
\text { Negative }\end{array}$ & $49(53.3 \%)$ & $30(56.6 \%)$ & $19(48.7 \%)$ & 0.454 \\
Positive & $43(46.7 \%)$ & $23(43.4 \%)$ & $20(51.3 \%)$ & 0.237 \\
CDX2 & & $5(6.5 \%)$ & $1(2.6 \%)$ & \\
Negative & $86(93.5 \%)$ & $48(90.6 \%)$ & $39(97.4 \%)$ & 0.833 \\
Positive & & $27(50.9 \%)$ & $19(48.7 \%)$ & \\
Mucin 5AC & $46(50.0 \%)$ & $26(49.1 \%)$ & $20(51.3 \%)$ & 0.376 \\
Negative & $46(50.0 \%)$ & & $23(59.0 \%)$ & \\
Positive & & $36(67.9 \%)$ & $16(41.0 \%)$ & \\
Mucin 2 & $59(64.1 \%)$ & $17(32.1 \%)$ &
\end{tabular}

SRC signet ring cell 
we might increase the application of safe and organ-preserving treatment to undifferentiated EGC patients.

In this study, $18.3 \%$ of cases had LNM. The LNM rate in EGC has been reported to be about $3.2 \%(0.0-20.3 \%)$ for mucosa-confined EGC and $19.2 \%(10.2-33.3 \%)$ for submucosa-invasive EGC $[27,28]$. In undifferentiated EGC, LNM occurs in 4.2-4.9\% of cases of mucosal EGC and $19.0-23.8 \%$ of cases of submucosa-invasive EGC $[5,6]$. The LNM rate in this study was consistent with the rates in previous reports. In our cohort, larger tumor size, presence of LVI, and mixed SRC carcinoma histologic type were associated with LNM. We did not observe any association between the well-known risk factors of depth of invasion or ulceration and LNM, possibly because we focused only on poorly cohesive, submucosa-invasive carcinomas.

The mixed SRC carcinoma group accounted for $43.5 \%$ of the cohort and was associated with a higher incidence of LNM and LVI. Intriguingly, intestinal metaplasia in the adjacent nonneoplastic mucosa was more frequent in the mixed SRC carcinoma subtype. This finding led us to speculate that intestinal metaplasia might indicate different histogenesis or carcinogenesis between the two types, and so we conducted immunoprofiling for lineage-associated markers, including mucin 2 and $\mathrm{CDX} 2$ as intestinal markers and mucin 5AC and mucin 6 as gastric markers. Our initial hypothesis was that pure SRC carcinoma might show a gastric phenotype, whereas mixed SRC carcinoma could be associated with an intestinal immunophenotype. However, we did not find differences in immunophenotypes between the two groups. CDX2 positivity was found in most $(93.5 \%)$ of the cases, and the proportion of mucin 5AC positivity between the two groups was nearly the same (49.1 and $51.3 \%$ for the pure SRC carcinoma group and the mixed SRC carcinoma group respectively). Furthermore, there were no differences in expression profiles between LNM-negative and LNM-positive cases. These four lineage-associated markers do not appear to be adequate for the evaluation of phenotypic differences between the two groups, although the lack of a difference could also be due to our limited sample size. More robust and comprehensive expression analysis such as DNA microarray or RNA sequencing could help further elucidate potential histogenetic differences between the two groups.

Although we focused on submucosa-invasive cancer in this study, Takizawa et al. [18] reported similar results for intramucosal EGC. They classified intramucosal EGCs into four types: pure differentiated (PD), pure undifferentiated (PU), mixed predominantly differentiated (MD), and mixed predominantly undifferentiated (MU). In their report, the incidences of LNM for PD, PU, MD, and MU EGC were $3.1 \%$ (4/130), $6.0 \%(11 / 184), 11.1 \%(6 / 54)$, and $19 \%$ $(8 / 42)$ respectively. The PU group showed a lower risk than the MD group. Because we studied only submucosa-invasive EGC, the difference in the incidence of LNM between the pure and mixed SRC carcinoma groups was more dramatic $(7.8 \%$ vs $31.9 \%)$ than that in the report of Takizawa et al. (6.0\% of PU EGCs vs $19 \%$ of MU EGCs). When we narrowed our cohort into a subgroup of consisting of tumors smaller than $2 \mathrm{~cm}$, no LVI, no ulceration, and less invasive than the SM2 level, then 64 cases (36 pure SRC carcinomas and 28 mixed SRC carcinomas) were included. The incidence of LNM in each subgroup was $2.8 \%(1 / 36)$ for pure SRC carconima and $10.7 \%(3 / 28)$ for mixed SRC carcinoma.

In conclusion, poorly cohesive EGC could be further classified by the purity of the poorly cohesive/SRC carcinoma component, and a pure SRC carcinoma subtype was associated with lower frequency of LVI, less frequent observation of intestinal metaplasia in the adjacent mucosa, and most importantly, a lower incidence of LNM. Furthermore, mixed SRC carcinoma histologic type was an independent risk factor for LNM in poorly cohesive, submucosa-invasive EGC. On the basis of our findings, we propose that when the diagnosis of SRC carcinoma is made, the pathology report should include the purity of the poorly cohesive/SRC carcinoma component or the presence of a minor tubular component, especially in cases of EGC, and such findings should be taken into consideration in the assessment of curable resection for ESD specimens.

Acknowledgments This research was supported by the Basic Science Research Program through the National Research Foundation of Korea, funded by the Ministry of Science, ICT and Future Planning (2012R1A1A1004403) and by a faculty research grant of Yonsei University College of Medicine for 2012 (6-2012-0044).

\section{Compliance with ethical standards}

Ethical standards All procedures were in accordance with the ethical standards of the responsible committee on human experimentation (Institutional Review Board of Yonsei University College of Medicine, approval no. 4-2014-0667) and with the Helsinki Declaration of 1964 and later versions. Informed consent or substitute for it was obtained from all patients for their being included in the study.

Conflict of interest The authors declare that they have no conflict of interest.

\section{References}

1. Gotoda T, Jung HY. Endoscopic resection (endoscopic mucosal resection/endoscopic submucosal dissection) for early gastric cancer. Dig Endosc. 2013;25(Suppl 1):55-63.

2. Gotoda T, Yamamoto H, Soetikno RM. Endoscopic submucosal dissection of early gastric cancer. J Gastroenterol. 2006;41:929-42.

3. Chung IK, Lee JH, Lee SH, Kim SJ, Cho JY, Cho WY, et al. Therapeutic outcomes in 1000 cases of endoscopic submucosal 
dissection for early gastric neoplasms: Korean ESD Study Group multicenter study. Gastrointest Endosc. 2009;69:1228-35.

4. Jung HY. Endoscopic resection for early gastric cancer: current status in Korea. Dig Endosc. 2012;24(Suppl 1):159-65.

5. Gotoda T, Yanagisawa A, Sasako M, Ono H, Nakanishi Y, Shimoda $\mathrm{T}$, et al. Incidence of lymph node metastasis from early gastric cancer: estimation with a large number of cases at two large centers. Gastric Cancer. 2000;3:219-25.

6. Hirasawa T, Gotoda T, Miyata S, Kato Y, Shimoda T, Taniguchi $\mathrm{H}$, et al. Incidence of lymph node metastasis and the feasibility of endoscopic resection for undifferentiated-type early gastric cancer. Gastric Cancer. 2009;12:148-52.

7. Hamilton SR, Aaltonen LA, editors. Pathology \& genetics. Tumours of the digestive system. Lyon: IARC Press; 2000.

8. Zhang M, Zhu G, Zhang H, Gao H, Xue Y. Clinicopathologic features of gastric carcinoma with signet ring cell histology. J Gastrointest Surg. 2010;14:601-6.

9. Chiu CT, Kuo CJ, Yeh TS, Hsu JT, Liu KH, Yeh CN, et al. Early signet ring cell gastric cancer. Dig Dis Sci. 2011;56:1749-56.

10. Kim DY, Park YK, Joo JK, Ryu SY, Kim YJ, Kim SK, et al. Clinicopathological characteristics of signet ring cell carcinoma of the stomach. ANZ J Surg. 2004;74:1060-4.

11. Huh CW, da Jung H, Kim JH, Lee YC, Kim H, Kim H, et al. Signet ring cell mixed histology may show more aggressive behavior than other histologies in early gastric cancer. J Surg Oncol. 2013;107:124-9.

12. Hyung WJ, Noh SH, Lee JH, Huh JJ, Lah KH, Choi SH, et al. Early gastric carcinoma with signet ring cell histology. Cancer. 2002;94:78-83.

13. Kunisaki C, Shimada H, Nomura M, Matsuda G, Otsuka Y, Akiyama $\mathrm{H}$. Therapeutic strategy for signet ring cell carcinoma of the stomach. Br J Surg. 2004;91:1319-24.

14. Yokota T, Kunii Y, Teshima S, Yamada Y, Saito T, Kikuchi S, et al. Signet ring cell carcinoma of the stomach: a clinicopathological comparison with the other histological types. Tohoku J Exp Med. 1998;186:121-30.

15. Gronnier C, Messager M, Robb WB, Thiebot T, Louis D, Luc G, et al. Is the negative prognostic impact of signet ring cell histology maintained in early gastric adenocarcinoma? Surgery. 2013;154:1093-9.

16. Chon HJ, Hyung WJ, Kim C, Park S, Kim JH, Park CH, et al. Differential prognostic implications of gastric signet ring cell carcinoma: stage adjusted analysis from a single high-volume center in Asia. Ann Surg. 2016. doi:10.1097/SLA. 0000000000001793.
17. Hanaoka N, Tanabe S, Mikami T, Okayasu I, Saigenji K. Mixedhistologic-type submucosal invasive gastric cancer as a risk factor for lymph node metastasis: feasibility of endoscopic submucosal dissection. Endoscopy. 2009;41:427-32.

18. Takizawa K, Ono H, Kakushima N, Tanaka M, Hasuike N, Matsubayashi $\mathrm{H}$, et al. Risk of lymph node metastases from intramucosal gastric cancer in relation to histological types: how to manage the mixed histological type for endoscopic submucosal dissection. Gastric Cancer. 2013;16:531-6.

19. Japanese Gastric Cancer Association. Japanese classification of gastric carcinoma: 3rd English edition. Gastric Cancer. 2011;14:101-12.

20. Tian MM, Zhao AL, Li ZW, Li JY. Phenotypic classification of gastric signet ring cell carcinoma and its relationship with clinicopathologic parameters and prognosis. World J Gastroenterol. 2007;13:3189-98.

21. Kim DH, Shin N, Kim GH, Song GA, Jeon TY, Kim DH, et al. Mucin expression in gastric cancer: reappraisal of its clinicopathologic and prognostic significance. Arch Pathol Lab Med. 2013; 137:1047-53.

22. Shim CN, Lee SK. Endoscopic submucosal dissection for undifferentiated-type early gastric cancer: do we have enough data to support this? World J Gastroenterol. 2014;20:3938-49.

23. Japanese Gastric Cancer Association. Japanese gastric cancer treatment guidelines 2010 (ver. 3). Gastric Cancer. 2011;14:113-23.

24. Kim YH, Kim JH, Kim H, Kim H, Lee YC, Lee SK, et al. Is the recent WHO histological classification for gastric cancer helpful for application to endoscopic resection? Gastric Cancer. 2016;19:869-875.

25. Park JH, Kim EK, Kim YH, Kim JH, Bae YS, Lee YC, et al. Epstein-Barr virus positivity, not mismatch repair-deficiency, is a favorable risk factor for lymph node metastasis in submucosainvasive early gastric cancer. Gastric Cancer. 2015. doi:10.1007/ s10120-015-0565-1.

26. Bosman FT, Carneiro F, Hruban RH, Theise ND, editors. WHO classification of tumours of the digestive system. Lyon: IARC Press; 2010.

27. Kwee RM, Kwee TC. Predicting lymph node status in early gastric cancer. Gastric Cancer. 2008;11:134-48.

28. Shin N, Jeon TY, Kim GH, Park DY. Unveiling lymph node metastasis in early gastric cancer. World J Gastroenterol. 2014;20:5389-95. 